\title{
Applications of Nanotechnology Towards Detection and Treatment of HIV/AIDs: A Review Article
}

\author{
Mope Edwin Malefane*
}

Nanotechnology and Water Sustainability Research Unit, University of South Africa, South Africa

ISSN: 2576-8840

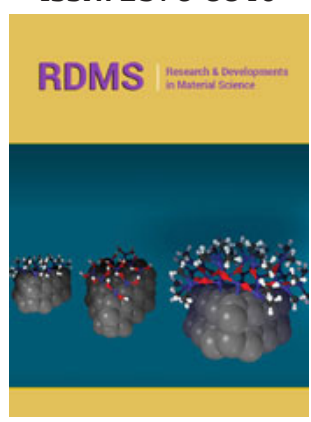

*Corresponding author: Mope Edwin Malefane, Nanotechnology and Water Sustainability Research Unit, College of Science, Engineering and Technology, University of South Africa, Florida 1709, South Africa

Submission: 眥 January 30, 2020

Published: 筒February 10, 2020

Volume 12 - Issue 5

How to cite this article: AMope Edwin Malefane. Applications of Nanotechnology Towards Detection and Treatment of HIV/ AIDs: A Review Article. Res Dev Material Sci. 12(5).RDMS.000796.2020.

DOI: $10.31031 /$ RDMS.2020.12.000796

Copyright@ Mope Edwin Malefane, This article is distributed under the terms of the Creative Commons Attribution 4.0 International License, which permits unrestricted use and redistribution provided that the original author and source are credited.

\begin{abstract}
There are frequent grumbles of HIV investigations that appears to be getting whisker close to a minor but influential word cure. Toxicity, viral reservoirs, societal discrimination and drug resistance increases the challenges faced with HIV detection and treatment. Nanotechnological applications in drug development and delivery promises to combat and correct issues associated with HIV treatment through generation of drugs with pharmacological advantages that are pioneered by unique materials properties at nanoscale compared to bulk, atomic scale or molecular dimensions. This mini review explores the profound research findings on different manipulations of nanosized materials (nanocapsules, nanocarriers, nanocrystals, etc.) towards rapid detection and cure and/or prevention of HIV. Moreover, possibilities for future investigations are suggested based on attained literature investigations and opinions as a roadmap towards successful HIV free generation and numerous advances in nanomedicine.
\end{abstract}

Keywords: Drug release; Early detection; HIV cure; Nanopharmaceuticals; Nanorobotics

\section{Highlights}

a) Roles of different stakeholders towards eradication of HIV/AIDS

b) Nanotechnology applications for possible anti-HIV remediation from infected individuals

c) Nanorobotics as an emerging possible solution to HIV epidemic

d) Challenges related to current methods of HIV treatment related to lack of knowledge and people's behaviour

e) Evolvement of Nano era from Macro and micro eras in biomedical applications

\section{Introduction}

There are numerous applications of nanotechnology since it was discovered that materials in nanoscale exhibited different yet fascinating properties to their molecular, atomic and bulk sized counterparts. The applications of nanotechnology range from novel generation of environmental pollution remediation [1,2], enhanced energy conversion and storage materials [3,4], technology advancement in agricultural [5], electronic and mining industries [6], electrochemical applications [7] and medicine [8]. All these innovative measures undertaken in numerous fields are intended to improve human health and socioeconomic standards for their disentanglement from complications that are encountered in the $21^{\text {st }}$ century.

Nanotechnology is often described as the synthesis and manipulation of materials with at least one dimension in the nanoscale or billionth of a meter $\left(10^{-9} \mathrm{~m}\right)[9-11]$. The applications of nanotechnology in medical applications stemmed from studies where the interaction of nanosized particles with biological systems was determined to be influenced by different physicochemical properties [11,12]. Like size exclusion related areas such as membrane technology [13], numerous biological spectacles (like passage across barriers and immune recognition) are ruled by size considerations $[8,14]$. The design and fabrication of nanoscale drugs with suitable dimensions may confer biological and physicochemical properties with a pharmacological superiority to conventional drug agents to address the challenges related to HIV detection and treatment if deliberately explored for these purposes [11]. Interestingly, 
numerous research has been done in this regard as the field of medical applications and investigations evolved from the Macro era that started in the mid-1960s where the centre of research was mostly polymers that were designed into macroscopic films, slabs, rods, spheres or rings, etc. to control the release of drugs by zero or nearly zero order kinetics $[11,15,16]$. The most prominent examples used ophthalmic polymer insert (Ocusert) to release pilocarpine over time and a rod-like progestasert that was used to release progesterone in 8 to 24 months while other investigations were conducted on transdermal drug deliveries by diffusion and partition $[8,11,17]$.

In the early 1970s, the micro era emerged as termed by Hoffman where biodegradable polymers were investigated based on their biodegradation kinetics for controlled drug release over time $[12,18]$. In this regard, poly (lactic-co-glycolic acid) (PLGA) gained enormous applications in implants due to its custom and biocompatible in vivo biodegradability. Moreover, the commercialization of PLGA occurred in mid-1980s with embedding of LHRH analogues in PLGA for treatment of prostate cancer as explained elsewhere [18]. The Macro and the micro eras were limited to the area of medicine involving drug release mechanisms based on different kinetics models [18-20]. Therefore, it was necessary to broaden the scope from just drug release to fabrication and development of novel materials towards the eradication of emerging diseases. This resulted in the emergence of the NANO era as termed by Hoffman, which is to date the most dynamic era since the pioneering studies in adjuvants (immunostimulants) at ETH Zurich by Speiser and his co-workers in mid-1970s even before the flashy hype about nanotechnology $[18,21]$. Since then, the potential of nano-sized materials with different shapes have increased from strength to strength as echoed by thousands of articles published per year to advance the NANO era from the research stage into commercialization [22] as few products have been developed already and are in the market place towards the treatment of some diseases but HIV $[18,23]$.

The focus of this mini review is to highlight examples and opinions on how nanopharmaceuticals as anti-HIV therapies for potential detection and cure of the disease could potentially be explored, the current findings as well as the numerous challenges related to application and approval of HIV/AIDS treatment. Generally, optimization of pharmacological characteristics of known antiretrovirals, delivery of antivirals to the immune system, brain or latent reservoirs, delivery of anti-HIV nucleic acids into diseaseridden cells and development of anti-HIV nanopharmaceuticals are some of the examples that will be discussed in detail. Moreover, some hurdles related to large scale and commercial applications of nanopharmaceuticals such as toxicity, massive cost of synthesis and unsolicited biological contacts will also be discussed.

\section{Nanopharmaceuticals}

Numerous investments are targeted at the development and enhancement of nanotechnology towards treatment of HIV/AIDS $[8,10,24,25]$. Nanomaterials such as dendrimers and many other inorganic nanoparticles (NPs) such as silver, gold, iron, and zinc can be used for HIV treatment by interfering at different stages in the life cycle of HIV and have generally been termed as nanopharmaceuticals $[25,26]$. A nanopharmaceutical represents any material in the nanorange with therapeutic potential [12]. Furthermore, NPs could act as convoys during drug delivery [27], intra-vaginal microbicides [28], adjuvants [25,28] and for the early detection of HIV-1 [25,2931]. Other materials that have been used in applications related to combating HIV/AIDS include polymeric nanoparticles, liposomes and micelles which have been used individually or with inorganic nanoparticles [32] or existing HIV conventional treatment agents to design nanopharmaceuticals $[33,34]$. The contribution of each form of material will be highlighted as this topic has been extensively discussed for detection and treatment of HIV [35-37].

Liposomes are nanocarriers (size between $25 \mathrm{~nm}$ - several microns) commonly used as drug delivery agents with an aqueous core engulfed inside a phospholipid bilayer for targeted drugs transfer to plasma proteins, reticuloendothelial system (RES) and phagocytic cells of infected patients' core [11,33]. RES acts as a reservoir for HIV replication while HIV is present in mononuclear phagocytic cells to improve the efficacy of anti-HIV drugs and reduce their side effects [11]. They are suitable antiretroviral agents as they become easily absorbed and excreted out of the body [25]. Polymeric NPs also have excellent uses for targeted drug delivery as they are stable, enhance sustainable drug release and are easily reproducible $[33,38]$. The explored polymeric nanoparticles for anti-HIV applications include poly(alkyl) cynoacrylates, poly(lactic acid) (PLA), poly(caprolactone), poly(lactic-co-glycolic acid) (PLGA), poly(ethylene glycol-co- (lactic-glycolic acid)) etc. with only PLA and PLGA approved for human use [37,39]. Therefore, biocompatible and biodegradable polymeric NPs were widely used as therapeutic carriers. Another interesting material employed for delivery of drugs are referred to as Micelles (size range from $10 \mathrm{~nm}$ to $100 \mathrm{~nm}$ in diameter) fabricated form encapsulating hydrophobic core inside a hydrophobic polymer have been explored as carriers for poorly soluble drugs $[12,18,39]$. Other drug delivery systems include dendrimers and solid lipid nanoparticles (SLNs) which goes to suggest that extensive discoveries and investigations are meant for improved delivery of drugs to specific targeted areas, but they also possess anti-HIV properties [8,33].

Inorganic nanoparticles with size ranging from (1-100nm) possess high loading efficiency with the common ones being gold nanoparticles (GNPs) that are studied as carriers and anti-HIV agents because their surfaces can be easily modified, biocompatible, excellent conductivity and surface plasmon, and easy reproducibility during synthesis $[8,40]$. Moreover, they could easily undergo surface modification through thiol linkages and possess other interesting photophysical, chemical and physical features for biomedical applications [39]. The Silver Nanoparticles (SNPs) have also been explored and their main route in action involves release of silver ions that enhance nucleic acid denaturation, cell membrane distortion, and antimicrobial activity for anti-HIV performance related mechanisms [33]. 


\section{Current HIV treatment}

There are more than 30 anti-retroviral drugs clinically accepted for application towards treatment of HIV-infected individuals that are broadly characterized as protease inhibitors (Lopinavir), CCR5 antagonists (Vicriviroc), fusion inhibitors (Enfuvirtide), integrase strand transfer inhibitors (Raltegravir), transcriptase inhibitors (Rilpivirine), non-nucleoside reverse transcriptase inhibitors (Nevirapine) and nucleoside/ nucleotide reverse transcriptase inhibitors (Lamivudine) with inability to eradicate viral reservoirs and only aiming at long term suppression of viral load instead of curing it $[11,14,28,41]$. They employ different mechanisms and detailed reviews on how they achieve viral inhibition with extensive examples in each class of drugs are provided elsewhere [14,28,39].

Despite the major evolution achieved in the last couple of decades towards inhibition of HIV-1 to prevent its clinical advancement to AIDS, the cure for this pandemic infection is yet to be realised [40]. High Activity Antiretroviral Therapy (HAART) which is a combination of at least 3 or more antiretroviral drugs was introduced and considered as the best option for treatment of patients infected with the virus irrespective of their age $[26,40]$ Long-acting inventions are acceptable tactics for applications in contraceptive cautionary medications and psychiatric disorders. Just recently, in vivo and in vitro studies were conducted without success in bioequivalence assay by Fandaruff et al. [26] which could be hardly explained by current technology when using a poorly soluble Efavirenz (EFV). However, different success stories had been reported for fabrication of long- acting formulations by incorporation of the active ingredient in an oil based suspension or solution, microfine watery suspension (steroid depots) or any suitable matrix (Risperidone ConstaÒ) for different applications including in HIV-1 studies [38,42].

Other examples that include the improvement of already available active agents for controlled release of drugs to specific target organs have been achieved successfully through the application and clinical testing of the in vitro with animals or in vivo results $[38,42,43]$. However, the toxicological risk and complex procedures required to ultimately find human volunteers to carry out the tests has hindered the commercialization of most medical related findings. For example, the use of TMC278 (rilpivirine) which is a derivative of poor oil and water soluble diaryl pyrimidine has been proved to interfere with HIV replication early inhibiting viral DNA synthesis and integration of the viral genome into the host DNA [42]. Moreover, it was determined to be efficient against a wide range of wild-type and HIV-mutant stains. Lastly; it was found to result in few metabolic and neuropsychiatric side effects than efavirenz as it was tolerable up to 48 weeks $[42,44]$. However, the detailed mechanistic investigations and related clinical testing procedures may require very expensive methodologies that could be possible with external funding.

Despite the widespread consensus that HAART is the best alternative method of treatment, its effectiveness maybe negatively affected by development of viral resistance. Incomplete HIV suppression and resistance to anti-viral medication may be triggered by factors of different origin that include non-compliance, drug resistance, insufficient drug potency, constrained tissue diffusion and the host related factors that are of genetic origin [11,40,41]. Therefore, compliance is critical towards successful administration and effectiveness of minimal inhibitory drug concentrations in the blood for understanding and examination of HIV or other chronic diseases relative suppression rates [44].

\section{Rapid detection of HIV/AIDS}

There are different groups that have investigated the possibility of rapid detection of HIV/AIDS than the currently used approaches as the delayed detection of the virus results in limitations towards treatment [45]. In this regard, gold nanoparticles (AuNPs) have been investigated extensively with promising results due to characteristics such as Surface Plasmon Resonance (SPR), Magnetic Properties (MRI), Surface Enhance Raman Scattering (SERS) and fluorescence behaviour that they display when conjugated with biocompatible and biological ligands. These properties find wide universality in biodiagnostics related to both diagnosis and therapy of cancer and HIV/AIDS that could be established from complete understanding of electrochemical methods, plasmon labelling and imaging and enzyme-linked immunosorbent assay (ELISA) [30]. Kumar et al. [30] gave a review on the roles that AuNPs have provided as a promising alternative towards the detection of cancer and HIV/AIDS [30]. The last 10 decades witnessed tremendous advancement of SPR and its use for therapy and diagnosis related to biomedical applications based on analysis of real-time kinetics of ligand-receptor interactions for detection of small molecules with modern spectroscopic advancement that enabled detections of single molecules in pico/nano concentrations. An interesting study was conducted by Tang et al. [9] where an AUNPs-Based biobarcode amplification assay (BCA) was determined to detect HIV-1 p24 antigen from a level as low as $0.1 \mathrm{pg} \mathrm{ml}^{-1}$ [9]. The importance of this study was in demonstration of low detection limit that can be achieved in the early stages of the viral infection.

The period between acquisition of HIV and seroconversion is close to 4 weeks and some methods that are currently used to detect the virus can give accurate results after 3 months of infection due to diverse genetic makeup of different individuals. The early detection of HIV is vital for protecting the wellbeing of people as there are progressive changes like formation of HIV reservoirs and duplication in the central nervous system after infection some of which are irreversible like demonising of CD4 lymphocytes $[41,46]$. Kosaka once said "The potential for HIV infectivity in the first stage of infection is much higher than in the later stages. Therefore, initiating antiretroviral therapy prior to seroconversion improves immune control and has been associated with benefits in CD4 cell count, a reduction in systemic inflammation, the preservation of cognitive function, and a reduction of the latent reservoir. Logically, its detection is critical to the prevention of HIV transmission" [46]. 
Therefore, a nanosensor that was developed by Kosaka et al. [46] which detected the virus in only one week of infection which is more rapid than the current available methods [46] would become an interesting aspect towards early determination of the virus.

Just recently, a graphene biosensor was developed for different applications such as HIV and cancer sensing capabilities [47] with a distinctive feature such as an ability to "visualize" molecular interactions in real time. The engineering of NPs in different directions and ability to tailor their physicochemical properties have given hope towards improving their robustness and binding affinities to various biomolecules and drug targets giving hope for treatment and detection of HIV with fabricated smart materials soon. The question remains to every researcher interested in the use of nanoparticles for sensory applications "How soon will these happen?"

\section{Nanopharmaceuticals with antiviral activity}

The area of nanomedicine that employs nanomaterials for diagnosis, prevention and treatment of diseases has proved that some nanomaterials have therapeutic effects and the interaction of biological systems and nanoscience is termed nanobiotechnology [12]. Several studies based on computational and experimental investigations have successfully identified compounds that could be applied to obstruct the replication of HIV capsid that was determined to be a target for specifically structured drugs to inhibit viral replication in vitro [28]. The various materials that have been determined to possess these special structural attributes in the nanoscale are dendrimers, fullerene (C-60) based structures and inorganic nanoparticles such as gold and silver in vitro $[11,33]$. However, there are still challenges and mechanisms that needs to be followed to achieve successful progressive investigations beyond in vitro studies that could lead to commercialization of these special materials but they have been illustrated to portray a potential for applications of therapeutic nanomaterials towards inhibition of HIV replication [40]. Other nanomaterials have been found to be extensively helpful towards improvement of already existing conventional anti-HIV agents or for transport of such materials to selected specific areas [37].

\section{Nanotechnology opportunities}

There are numerous investigations and opinions that are reported in the last decades that are concentrated on the different in-depth research and studies based on expert knowledge and understanding. Opportunities that are discussed in this mini review will only explore the mechanisms that have been tested towards battling HIV/AIDS and more research-based opinions that could positively augment humanity towards its complete eradication.

The most recent example of an alternative approach is immunotherapy that controls the immune response to counter HIV $[8,40]$. The mechanism involved in CD8+ cytotoxic T-cell responses to acute HIV infection seem to be ordinary and neutralization of antibodies production by B cells is absent or deferred [40].
However, with progression of time, viral mutations result in depletion of CD8+ T cell cytotoxic performance. Since the major effects of HIV infections leads to depletion of CD4+ T cells that are normally referred to helper cells that are accountable for assisting in supportive functions [28]. The loss of these cells leads to profound immunosuppression that is triggered by non-functional B cells, natural killer cells and macrophages in patients with chronic HIV infections [29,48]. Draz et al. [49] integrated cellphone-based optical sensing, loop-mediated isothermal DNA amplification and micromotor motion for molecular detection of HIV-1 [49]. In recent years, there has been increasing interest in the therapeutic use of immune responses and this filed is expected to grow exponentially provided enough volunteers are successfully found for both donations and testing purposes [40].

The recent emergence of nanorobots is an interesting field towards controlled and sophisticated eradication of HIV/AIDS and the whole area of nanomedicine for regulation and treatment of other diseases such as cancer. Theses nanorobots provides a very interesting perspective when combined with nanosponges that captivate toxins giving hope that a system could exist someday that would also be able to not only destroy but extract and excrete the toxins and viruses out of the body by creation of tiny robots that could proficiently wash out detrimental constituents that leads to diseases in the body $[10,50]$. Joshi et al. [10] theoretically investigated a specific treatment of nanorobots incorporated with nanosensors to sense AIDS infected WBCs and control the level of WBCs in the blood stream to stimulate an HIV infected patients' immune system to be less vulnerable to diseases [10].

Nanoparticles have a huge potential as adjuvants and delivery systems for vaccines and have been employed as it has happened over the past few decades for controlled release of various agents $[51,52]$. This has prodigious compensations for vaccine delivery because the controlled release of antigens could result to a continued and tougher initiation of the immune response for HIV related illness intercession [40]. Martins et al. [53] explored efavirenz (EFV) loaded poly(lactic-co-glycolic) acid (PLGA) NPs targeted to the blood-brain barrier (BBB) towards HIV neuropathology treatment with sustained in vitro release of $50 \%$ for $24 \mathrm{~h}$ and its permeability surpassed that of the free EFV by a magnitude of 1-3 fold [53]. A review of biodegradable polymers by Amsden examines new polymer biomaterials that have been established to advance distribution of active agents [36].

Other novel findings that still need to be investigated include a bee venom loaded nanoparticles that were reported to selectively destroy HIV without affecting neighbouring cells [54]. Moreover, it was recently suggested that fabrication and manipulation of aluminium nanorobots could modify the human immune system from its reactive state to an adaptive state which could results in improved response towards cancer, HIV and other age-related degenerative ailments $[10,50]$. Despite the field of nanorobots being in its infant stages, there is inspiring progression in the field 
with reassuring opportunities that produced hopes for curing many diseases including HIV/AIDS [50] and easy surgical events that do not necessarily involve doctors having to undergo surgical procedures.

Other interesting materials referred to as multifunctional carriers have been explored and despite confirmed challenges with optimization and quality assurance restrictions, they may become an interesting field to explore towards eradication of HIV/ AIDS pandemic [18]. Therefore, it can be inferred that there is hope even though it may require several decades for the required breakthrough to eliminate HIV virus from infected individuals.

\section{Limitations}

There are several factors associated to limitations of either finding a cure for HIV or implementation of nanotechnology towards successfully eradicating the virus from blood streams of infected patients. Most challenges are related to available technological knowledge and others are related to limitations based on legislative and behavioural normalities of different stakeholders.

Prof. Françoise Barré-Sinoussi said in her interview, "The genetic variability of HIV is one obstacle, another is the way the virus stores itself in 'reservoirs' such as the lymph nodes of the intestinal region. Eradication will not be easy because the virus stays in these 'reservoirs' and is not affected by the immune response, even after 10 years of antiretroviral treatment [23]." There are two types of viral reservoirs existing in tissues like the anatomical reservoirs that are remotely located for access by antiviral optimal dosages due to blood-cerebrospinal fluid barrier, BBB and bloodtestes barrier $[37,55]$. The others include cellular reservoirs that encourages resistance of latently arranged viruses to antiviral activity due to efflux proteins such as P-glycoprotein and multidrug resistance protein on the cell surface preventing the drugs from reaching therapeutic intracellular concentrations $[37,55]$.

Other interesting limitations for current treatment of HIV under clinically proven drugs is the emergence of drug resistance that can be triggered by individual's genetic makeup or unfaithfulness towards prescribed treatment [56]. The latter is another aspect of people behaviour towards acceptance of HIV treatment [57]. While in most developing countries, particularly in rural areas, the behaviour of health care professionals towards HIV infected patients and the nature of the lack of community knowledge towards HIV/ AIDS has often seen numerous people entrapped with resistance to antivirals [58]. Lastly, the numerous steps that are required for novel nano-medication to pass clinical and toxicological tests [32] has often resulted in different delays towards potentially promising ideas being implemented.

\section{Conclusion}

In a nutshell, there is a vivid message for policy makers, HIV infected individuals, academic researchers, and everyone interested in the eradication of HIV epidemic that if it were to be possible, the cure for HIV relies in extensive exploration and applications of nanopharmaceuticals and may involve numerous sacrifices to be successfully attained. The role that would be played by investors and volunteering individuals towards discovery, testing and implementation of nanopharmaceuticals would answer the question, "After how many decades until a cure to HIV/AIDS will be achieved?" Therefore, everyone has a strong role to play towards global extermination of HIV/AIDS.

\section{Acknowledgement}

This work was not financially supported by any institution or organization.

\section{References}

1. Lin Y, Mehrvar M (2018) Photocatalytic treatment of an actual confectionery wastewater using $\mathrm{Ag} / \mathrm{TiO}_{2} / \mathrm{Fe}_{2} \mathrm{O}_{3}$ : Optimization of photocatalytic reactions using surface response methodology. Catalysts 8: 409-425.

2. Malefane ME, Feleni U, Kuvarega AT (2019) A tetraphenylporphyrin/ $\mathrm{WO}_{3}$ /exfoliated graphite nanocomposite for the photocatalytic degradation of an acid dye under visible light irradiation. New J Chem 43: 11348-11362.

3. Cataldi P, Athanassiou A, Bayer I (2018) Graphene nanoplatelets-based advanced materials and recent progress in sustainable applications. Appl Sci 8: 1438.

4. Collins G, Armstrong E, Mcnulty D, Hanlon SO, Geaney H, et al. (2016) 2D and 3D photonic crystal materials for photocatalysis and electrochemical energy storage and conversion. Sci Technol Adv Mater 17: 563-582.

5. Hirakawa H, Hashimoto M, Shiraishi Y, Hirai T (2017) Photocatalytic conversion of nitrogen to ammonia with water on surface oxygen vacancies of titanium dioxide. J Am Chem Soc 139(31): 10929-10936.

6. Jurow M, Schuckman AE, Batteas JD, Drain CM (2010) Porphyrins as molecular electronic components of functional devices. Coord Chem Rev 254: 2297-2310.

7. Mafa PJ, Kuvarega AT, Mamba BB, Ntsendwana B (2019) Photoelectrocatalytic degradation of sulfamethoxazole on g-C $3 \mathrm{~N} 4$ / $\mathrm{BiOI} / \mathrm{EG}$ p-n heterojunction photoanode under visible light irradiation. Appl Surf Sci 483: 506-520.

8. Cao S, Woodrow KA (2019) Nanotechnology approaches to eradicating HIV reservoirs. Eur J Pharm Biopharm 138: 48-63.

9. Tang SX, Hewlett I (2010) Nanoparticle-based immunoas- says for sensitive and early detection of HIV-1 capsid (p24) antigen. J Infect Dis 201: 59-64.

10. Joshi A, Pardeshi A (2012) Anti-HIV using nanorobots. IOSR J Electr Electron Eng 7(6): 84-90.

11. Parboosing R, Maguire GEM, Govender P, Kruger HG (2012) Nanotechnology and the treatment of HIV infection. Viruses 4: 488-520.

12. Singh L, Kruger HG, Maguire GEM, Govender T, Parboosing R (2017) The role of nanotechnology in the treatment of viral infections. Ther Adv Infect Dis 4(4): 105-131.

13. Kumar S, Nandi BK, Guria C, Mandal A (2017) Oil removal from produced water by ultrafiltration using polysulfone membrane. Brazilian J Chem Eng 34: 583-596.

14. Curley P, Liptrott NJ, Owen A (2018) Advances in nanomedicine drug delivery applications for HIV therapy. Futur Sci OA 4: FS0230.

15. Ramanathan K, Avnir D, Modestov A, Lev $O$ (1997) Sol-Gel derived ormosil-exfoliated graphite- $\mathrm{TiO}_{2}$ composite floating catalyst: Photodeposition of copper. Chem Mater 9: 2533-2540. 
16. Kanakaraju D, Glass BD, Oelgemo M (2014) Titanium dioxide photocatalysis for pharmaceutical wastewater treatment Titanium dioxide photocatalysis for pharmaceutical wastewater treatment. Env Chem Lett 12: 27-47.

17. Kroon M (2015) Recognising and managing increased HIV transmission risk in newborns. South Afr J HIV Med 16(1): 1-7.

18. Merkle HP (2015) Drug delivery's quest for polymers: Where are the frontiers? Dedicated to Robert Gurny on the occasion of his $70^{\text {th }}$ birthday. Eur J Pharm Biopharm 97: 293-303.

19. Chong MN, Jin B, Chow CWK, Saint C (2010) Recent developments in photocatalytic water treatment technology: A review. Water Res 44(10): 2997-3027.

20. Sharma AK, Tiwari RK, Gaur MS (2012) Nanophotocatalytic UV degradation system for organophosphorus pesticides in water samples and analysis by Kubista model. Arab J Chem 9(2): 1755-1764.

21. Safari NJ, Zarnegar Z(2014) Advanced drug delivery systems: nanotechnology of health design A review. J Saudi Chem Soc 18(2): 8599.

22. Angel M, Machuca-martinez F, Li G (2013) Effective quantum yield and reaction rate model for evaluation of photocatalytic degradation of water contaminants in heterogeneous pilot-scale solar photoreactors. Chem Eng J 215-216: 937-947.

23. Fleck F (2009) A significant discovery, now a dream for a cure. An interview with Françoise Barré-Sinoussi. Bull World Health Organ 87: 10-11.

24. Cuffari B (2017) Nanotechnology and the fight against HIV.

25. Saravanan M, Asmalash T, Gebrekidan A, Gebreegziabiher D, Araya T, et al. (2018) Nano-medicine as a newly emerging approach to combat Human Immunodeficiency Virus (HIV). Pharm Nanotechnol 6: 17-27.

26. Fandaruff C, Segatto Silva MA, Galindo Bedor DC, De Santana DP, Rocha HVA, et al. (2015) Correlation between microstructure and bioequivalence in Anti-HIV Drug Efavirenz. Eur J Pharm Biopharm 91: 52-58.

27. Heon Lee I, Palombo MS, Zhang X, Szekely Z, Sinko PJ (2019) Design and evaluation of a CXCR4 targeting peptide 4DV3 as an HIV entry inhibitor and a ligand for targeted drug delivery. Eur J Pharm Biopharm 138: 1122.

28. Kim PS, Read SW (2010) Nanotechnology and HIV: Potential applications for treatment and prevention. Wiley Interdiscip Rev Nanomedicine Nanobiotechnology 2(6): 693-702.

29. Gao Y, Kraft JC, Yu D, Ho RJY (2019) Recent developments of nanotherapeutics for targeted and long-acting, combination HIV chemotherapy. Eur J Pharm Biopharm 138: 75-91.

30. Kumar A, Mazinder-Boruah B, Liang (2011) Gold nanoparticles: Promising nanomaterials for the diagnosis of cancer and HIV/AIDS. J Nanomater 2011: 202187.

31. Klouda L (2015) Thermoresponsive hydrogels in biomedical applications A seven-year update. Eur J Pharm Biopharm 97: 338-349.

32. Frazer RA (2012) Use of silver nanoparticles in HIV treatment protocols: A research proposal. J Nanomedicine Nanotechnol 3: 1-5.

33. Kumar L, Verma S, Prasad DN, Bhardwaj A, Vaidya B (2015) Nanotechnology: A magic bullet for HIV AIDS treatment. Artif Cells Nanomedicine Biotechnol 43(2): 71-86.

34. Ramana LN, Sharma S, Sethuraman S, Ranga U, Krishnan UM (2015) Stealth anti-CD4 conjugated immunoliposomes with dual antiretroviral drugs - Modern Trojan horses to combat HIV. Eur J Pharm Biopharm 89: 300-311. 35.https: / / aidsinfo.nih.gov/contentfiles /lvguidelines / adultandadolescentgl.pdf

36. Amsden B (2015) Novel biodegradable polymers for local growth factor delivery. Eur J Pharm Biopharm 97: 318-328.

37. Lenjisa JL, Woldu MA, Satessa GD (2014) New hope for eradication of HIV from the body: The role of polymeric nanomedicines in HIV/AIDS pharmacotherapy. J Nanobiotechnology 12: 4-9.

38. Ariza-Sáenz M, Espina M, Bolaños N, Calpena AC, Gomara MJ, et al. (2017) Penetration of polymeric nanoparticles loaded with an HIV-1 inhibitor peptide derived from GB virus $\mathrm{C}$ in a vaginal mucosa model. Eur J Pharm Biopharm 120: 98-106.

39. Victor OB (2019) Nanoparticles and Its Implications in HIV/AIDS Therapy. Curr Drug Discov Technol.

40. Mamo T, Moseman EA, Kolishetti N, C Salvador, Shi J, et al. (2010) Emerging nanotechnology approaches for HIV/AIDS treatment and Prevention. Nanomedicine (Lond) 5(2): 269-285.

41. Esté JA, Cihlar T (2010) Current status and challenges of antiretroviral research and therapy. Antivir Res 85(1): 25-33.

42. Van Gyseghem E, Pendela M, Baert L, Rosier J, De Man H, et al. (2008) Powder for reconstitution of the anti-HIV-1 drug TMC278 - Formulation development, stability and animal studies. Eur J Pharm Biopharm 70(3): 853-860.

43. Schneider EL, Ashley GW, Dillen L, Stoops B, Austin NE, et al. (2015) Halflife extension of the HIV-fusion inhibitor peptide TRI-1144 using a novel linker technology. Eur J Pharm Biopharm 93: 254-259.

44. Baert L, van 't Klooster G, Dries W, François M, Wouters A, Basstanie E, et al. (2009) Development of a long-acting injectable formulation with nanoparticles of rilpivirine (TMC278) for HIV treatment. Eur J Pharm Biopharm 72(3): 502-508.

45. Marsden MD, Zack JA (2009) Eradication of HIV: Current challenges and new directions. J Antimicrob Chemother 63: 7-10.

46. Kosaka PM, Pini V, Ruz J, Da Silva R, González M, et al. (2014) Detection of cancer biomarkers in serum using a hybrid mechanical and optoplasmonic nanosensor. Nat Nanotechnol 9: 1047-1053.

47. Stebunov Y, Materials ACSA, Volkov V, Denmark S, Aftenieva O (2015) Highly sensitive and selective sensor chips with graphene-oxide linking layer. ACS Appl Mater Interfaces7(9): 21727-21737.

48. Ricciotti E, Fitz Gerald GA (2012) Prostaglandins and Inflammation. Arter Thromb Vasc Biol 31(5): 986-1000.

49. Draz MS, Kochehbyoki KM, Vasan A, Battalapalli D, Sreeram A, et al. (2018) DNA engineered micromotors powered by metal nanoparticles for motion-based cellphone diagnostics. Nat Commun 9: 4282-4294.

50. Li S, Jiang Q, Liu S, Zhang Y, Tian Y, et al. (2018) A DNA nanorobot functions as a cancer therapeutic in response to a molecular trigger in vivo. Nat Biotechnol 36(3): 258-264.

51. Nouailhat A (2006) An introduction to nanoscience and nanotechnology. Introd Aux Nanosci Aux Nanotechnologies, ISTE, John Wiley \& Sons, London, UK, pp. 1-229.

52. Mauro M, Crosera M, Pelin M, Florio C, Bellomo F, et al. (2015) Cobalt oxide nanoparticles: Behavior towards intact and impaired human skin and keratinocytes toxicity. Int J Environ Res Public Health 12(7): 82638280 .

53. Martins C, Araújo F, Gomes MJ, Fernandes C, Nunes R, et al. (2019) Using microfluidic platforms to develop CNS-targeted polymeric nanoparticles for HIV therapy. Eur J Pharm Biopharm 138: 111-124.

54. AZoNano (2014) Using nanotechnology in HIV Treatment. 
55. Agency USEP, Davis J, Wang A, Shtakin J (2010) Nanomaterial case studies: Nanoscale titanium dioxide in water treatment and in topical sunscreen. US EPA Res Triangle Park, pp. 13-14.

56. Rossouw TM, van Dyk G, van Zyl G (2019) Rapid emergence of resistance to antiretroviral treatment after undisclosed prior exposure: A case report. South Afr J HIV Med 20: 3-5.
57. Reif LK, Rivera V, Bertrand R, Belizaire M, Joseph B, et al. (2019) "FANMI": A promising differentiated model of HIV care for adolescents in haiti. J Acquir Immune Defic Syndr 82(1): 11-13.

58. (2015) Ministry of health Lesotho, global aids response progress report 2015 Lesotho country report, Follow-Up to the 2011 political declaration on HIV/AIDS: Intensifying efforts to eliminate HIV/AIDS.

For possible submissions Click below: 